\title{
Acceptability to parents of a baby-led approach to introducing solids
}

\section{Abstract}

In Baby-Led Weaning (BLW), infants are offered foods they can pick up and feed themselves from the start of complementary feeding. Infants who are fully BLW are not spoonfed at all by their parents, feeding themselves all their foods instead. The Baby-Led Introduction to SolidS (BLISS) study was a randomised controlled trial of the effect of a modified version of BLW5 on infant growth, iron status, and risk of choking, and provides an opportunity to investigate parents' experiences of using a baby-led approach to infant feeding. Complementary feeding methods are usually chosen by parents, so it is important to ascertain whether parents find a baby-led method of introducing solids acceptable if they are assigned to follow it. This is both to determine whether it would be feasible to randomise them to follow BLW in future randomised controlled trials and because, if beneficial effects of BLW are shown, policy makers need to know whether parents would find it acceptable to follow BLW. The aim of this analysis was to determine the acceptability to parents of a baby-led approach to complementary feeding when their infant was 7 to 12 months of age. In total, 206 participants were randomised to Control $(n=101)$ or BLISS $(n=105)$ groups in the third trimester of pregnancy. When the infants were $7,8,9$, and 12 months of age, questionnaires were administered to determine parents' happiness and frustration with their feeding method, and attitudes regarding its convenience, mess, and expense. Food cost was estimated using supermarket prices linked to a 3-day weighed diet record collected at 7 months of age. Both groups reported high levels of happiness and convenience, but also reported finding complementary feeding very frustrating. There were two significant differences between the groups the BLISS group reported less messiness, and were more likely to perceive their method as expensive. The actual food cost per day was not statistically significantly different between the two groups (NZ\$1.70 for BLISS, NZ\$1.90 for Controls). In conclusion, parents did not find a baby-led approach to introducing solids any less acceptable than control parents found standard infant feeding. It is, therefore, feasible to run studies where parents are randomised to follow a baby-led approach to complementary feeding and, should health advantages to BLW be identified, parents are likely to find BLW acceptable to follow.

\section{Conflict of Interest}

There is no conflict of interest. 